\title{
On the Current Status and Development of Lectures of Second Classroom —illustrated by the case of universities of finance and economics
}

\author{
Meihui ${ }^{1, a}$ \\ ${ }^{1}$ Capital University of Economics and Business Students' affair Office, Fengtai, Beijing, China \\ a631773166@qq.com
}

Key words: Second classroom, lecture, quality education, universities of finance and economics.

\begin{abstract}
As an important carrier for all-round promotion of quality education, the second classroom of college students has effectively widened the content of classroom education, and the knowledge imparted in the form of lectures can cover more students, and the knowledge can be sorted out in a more comprehensive and systematic way. Moreover, the inherent basic knowledge on the textbooks can be extended to the dominant theme education, the edification of art and culture, and the free talks on essays and anecdotes. However, all colleges and universities have encountered the problems in low attendance rate of lectures, low rise rate, and the difficulty to achieve the expected effects. This paper combines questionnaire survey and individual interviews of students of universities of finance and economics to understand the reasons for the questions and puts forward corresponding suggestions so as to create a "cost-effective" lecture mode in the second classroom.
\end{abstract}

\section{Research summary}

This year, on the eve of the arrival of "Youth Day" and the 120th anniversary of the founding of Peking University, General Secretary Xi Jinping visited Peking University, held a discussion with the students and teachers of Peking University and delivered a keynote address. He pointed out that our future requires the young people to continue to struggle. A booming education leads to a booming country and a strong education leads to a strong country. The "13th Five-year Plan" has proposed to improve the quality of education in an all-round way, to perfect the quality assurance system of higher education, to reform the training mechanism of talents, and to put forth efforts to cultivate the students' sense of social responsibility, spirit of innovation and practical ability. After entering the twenty-first century, it's the objective requirement for the colleges and universities to better fulfill the basic functions of personnel training to cultivate the professional talents with good comprehensive qualities and abilities ${ }^{1}$. Throughout the development and the mission of the university, the university not only has the impact of curriculum culture, which occupies a dominant position, on the students, but there is also an important form of education which has been playing an important role in the training of talents, that is, college lectures ${ }^{2}$. As the main manifestation of the second classroom, the lecture is the thrust to improve the students' soft power, and how to maximize the benefit of the students and lectures still remains to be thought and explored.

By combining the release of online questionnaires and visits to the lectures organizers of universities of finance and economics, the author has a preliminary understanding of the types of college students' reference for the lectures, the frequency of attending lectures, the assistance of lectures in growth, the channels for the information of the lecture, the opinions on the present lectures and so on, and makes a brief summary of the frequency and purpose of the students' participation in the lectures, and their expectations and suggestions of the lectures and so on, as well as analyzes the current situation of the college lectures. 


\section{Analysis of current situation}

\subsection{Obstacles to "killing two birds with one stone": Lectures are difficult to take account of both students' interests and training objectives.}

Through the questionnaire survey, the students' interest in lectures is obtained, as shown in the following figure. The three most preferred types of lectures are career planning, psychological lectures and the latest international situation. For example, the students should improve their abilities of employment so as to adapt to the society ${ }^{3}$. Lectures in career planning are of the utmost importance in job hunting. At the same time, we also saw the periodicity in the development of interest. Most junior students who have just entered the university tend to choose lectures on career planning, and the senior students who are graduating tend to take a lecture on the entrance exams for postgraduate schools and choice of occupation.

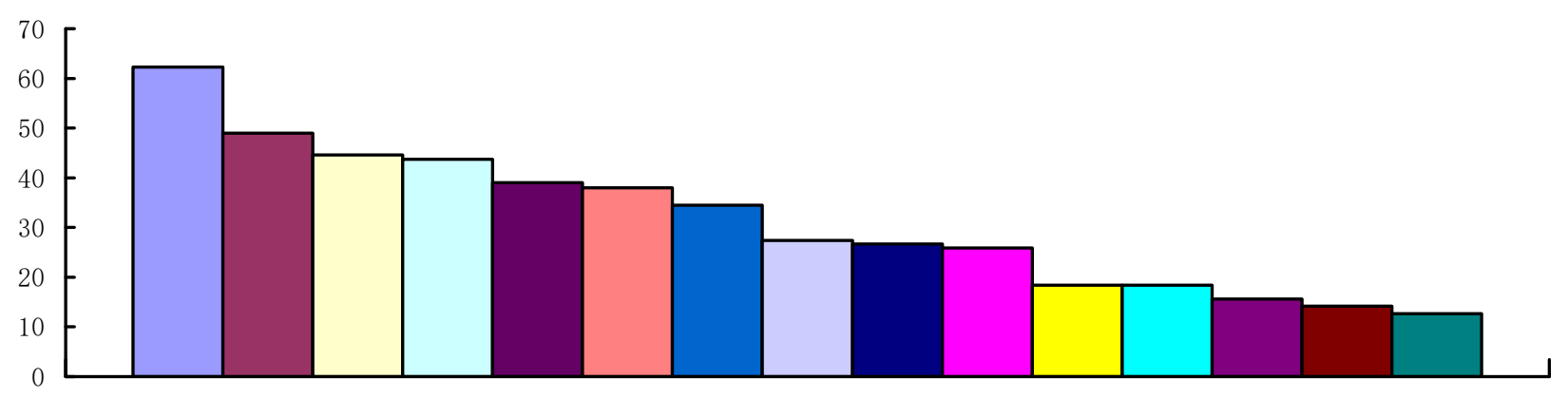

\begin{tabular}{|ll|}
\hline$\square$ Career planning & $\square$ Skills of mental adjustment \\
$\square$ Love psychology & $\square$ International situation \\
$\square$ Entrepreneurial skills & $\square$ Courses on stress management \\
$\square$ Contemporary literature & $\square$ Characters and stories \\
$\square$ Moral cultivation & $\square$ Culture of ancient Chinese poetry \\
$\square$ Traditional opera and calligraphy and painting & $\square$ Construction of dormitory relations \\
$\square$ Ancient architecture & $\square$ Military exploration \\
$\square$ Issue of sovereignty & \\
\hline
\end{tabular}

Figure 1: Types of lectures that are hoped to be provided by the university

At present, the current situation of lectures in universities is that it is vulnerable to students' "cold reception" and that it is formally oriented ${ }^{4}$. Meanwhile, the subjective ideas of the organizers for the lectures are out of line with the actual needs of the students, and the diversity of entertainment and learning methods have made most of the students feel reluctant to take one or two hours to listen to a lecture that is far away from their ideal. On the one hand, in terms of the public lectures with "celebrity effect", the theme is popular and easy to understand, and the keynote speaker tends to be so amiable that the students always attend such lectures without being asked. On the other hand, the academic lectures have made students at a distance from them, and they are not willing to spend more time on learning and thinking about the research issues that are not their major or that they are not interested in. Not to mention lectures on theme education, ideology and so on, there is no lack of compulsory participation.

\section{2 "Difficult problems" for the post-1995 generation: Utilitarian learning, quick marketing and too much fragment information}

Despite the fact that there have been the post-2000 generations of college students on campus, most of the groups and leading forces are still "the post-1995 generation", whose interest in learning may be easily worn out by bad learning habits and learning attitudes. Sometimes the school is holding a win-win lecture of students' growth and interest, but the current state of college students has made it difficult for the college lectures to play a real effect, so that it fails to reach the original intention 
of the lecture itself.

After college students enter the university, their interest in lectures tend to have a marginal diminishing effect. The survey found that most of the students show a strong interest in lectures and they are able to listen carefully to the lectures when they just enter the university, which is because that these lectures will be of great help to the students that have just entered the university. However, when it comes to the sophomore and junior years, the students' expectation and interest in the lecture will get lower and lower, and they will attend the lectures less and less, which is because of the fact that lectures have been solidified in their mind and that they hold that the lectures have followed the same pattern.

Table 1: Is the lecture useful to you?

\begin{tabular}{|c|c|}
\hline Option & Proportion \\
\hline Yes & $16.04 \%$ \\
\hline No & $10.38 \%$ \\
\hline Most of them are useful & $36.79 \%$ \\
\hline Most of them are useless & $36.79 \%$ \\
\hline
\end{tabular}

Students in universities of finance and economics are suffering less pressures of scientific research as well as more stress of practical activities. Some college students generally have the learning habits of fast selling, and they tend to be utilitarian in choosing lectures, while most of their motivation comes from obtaining the corresponding credits and rewards, rather than learning some real knowledge and skills. Such a utilitarian thought is likely to be affected by the learning atmosphere of students. Some lectures are able to arouse the interest of the students, and there are some substantial contents so that the students are very fond of attending such lectures, but the negative atmosphere around the students have made them gradually lose their original interest in the lecture. At the same time, despite the fact that some students prefer interactive lectures, part of the students are ashamed to participate in such lectures, while they only hope to listen to the lectures quietly. It is obvious that the students' psychology of attending lectures is somewhat contradictory.

\subsection{Mixture of high-quality lectures and poor-quality lectures: It is difficult to guarantee the time utilization rate.}

Knowledge is applied, tought and learnt by person which push the "mankind" into the middle of the knowledge society. ${ }^{5}$ The organizers have been carefully selecting the keynote speaker every time, but there are still a number of factors that can not be controlled. For example, some keynote speakers, because of the urgent time, may regard their assistant as a "ghost writer", but they fail to be well prepared, which means that the organizers are unable to control the attention of the keynote speaker to the lecture. Moreover, sometimes there may be a mismatch between the topic of the keynote speaker and the expected topic, and even the keynote speaker may go off the topic or change the topic at the last minute. However, even if the topic of the keynote speaker is as expected, the content or form of the lectures may not be able to achieve the desired result. 

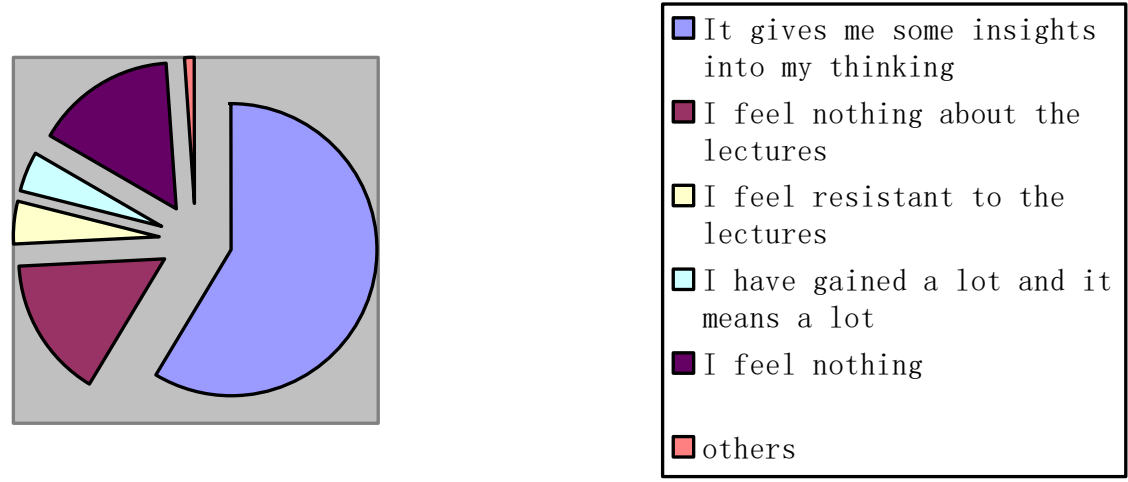

Figure 2: Feelings about the previous lectures

Most of the lectures in colleges and universities are widely applicable but relatively boring lectures, with a lack of personalized, accurate and infectious lectures. Some lectures are "short of useful content", or the theories of some lectures are out of line with the practical situation, and there is only some theoretical knowledge, but it is lacking in concrete methods that can be used in real life. In this case, students may feel their heads in the clouds, and they are unable to find any method to put it into practice. One of the important reasons for the absence of the ideological and political lectures on campus is that it is difficult to find a unique and well received orientation among students. ${ }^{6}$

Finally, students associations, colleges, departments, schools and other organizations all have the right to host, but it is difficult to take the unified standards. Some keynote speakers spout eloquent speeches, but they have no interaction with the students. Some speakers are able to arouse students' enthusiasm in spite of the shortage of theoretical discourse. Besides, since some speakers lack personal charm, students are unable to concentrate on the lecture. For students, they can not accept the fact that they are always "dragged" to the lectures ${ }^{7}$, since some of the lectures themselves are part of the sponsorship to students' activities, and there is no lack of some special words, such as "a click-bait title", "credit award" and "compulsory requirement".

\subsection{Inactive role as a go-between: Imperfect channels of resource sharing and improper propaganda work}

University culture has four characteristics, that is, culture, education, times and high grade ${ }^{8}$ Therefore, universities have the ability and the required soil to cultivate outstanding talents as pillars of the state, and are rich in top resources in various professional fields. But how to apply the resources to the process of students' growth and development requires a lot of sound measures and perfect systems.

According to the survey data, students of universities of finance and economics are more inclined to the lectures in forms of practice and salon, that is, interactive and highly experiential lectures. Such lectures often involve a small number of spectators, and it is a "small classroom" compared with the large-scale lectures involving hundreds of spectators. But it takes a very high cost to hold such a lecture. Therefore, it is quite difficult to find proper ways to make use of the existing resources to reduce lecture costs and to meet the students' preferences. Colleges and universities are facing a shortage of docking between colleges and schools. On the one hand, the school lectures are oriented to the students in the whole school. They tend to give priority to general education, such as professional career planning, dominant theme education, psychological knowledge, excellent traditional Chinese culture, talks by famous scholars and experts, entrepreneurial knowledge and other highly universal lectures. On the other hand, the contents of the lectures in the colleges and departments are biased towards the majors of the college, which lack certain universality. However, a large number of students have a strong interest in professional or academic lectures, such as law education, English language and literature, human resources and other topics, which are all favored by a great many students. 
The propaganda prior to the lecture will help students to understand the content, time and place of the lecture. What's more, the simple, direct and exciting way of publicity will greatly increase the attendance rate of the lecture. The propaganda work during the lecture will be conducive for the students that participate in the lecture to enhancing their sense of achievement and superiority, and guiding them to focus on such topics and the lectures of similar types in the future. The effective propaganda after the lecture is better to break the situation of the information asymmetry. In case that the students attend the lectures in a passive manner, their concern about the content of this lecture is bound to be far less than that when they take the initiative to obtain the content. In case that the lecture is by the keynote speaker that students are interested in or on the topic that they are interested in, they are likely to have access to the relevant information on the Internet prior to attending the lecture, so that they will listen more carefully to the lecture by the keynote speaker, and have certain resonance with the speaker.

\section{Relevant suggestions}

In view of the problems mentioned above, the author thinks that the following aspects should be improved, so as to enhance the "cost performance" of the lecture.

\subsection{To enhance supply-side structural reform of lectures based on data}

Only with the support of clear and reliable data, the lectures that are well received by students can be held with great facility. Prior to the lecture, a survey may be carried out to learn about students' urgent needs in the near future, which can be combined with the late feedback of the lecture to form a system. On the one hand, the establishment of a phased real-time survey on the system is conducive to the effective implementation of the lecture and assists the organizers in knowing the immediate effect of the lecture. For most people, they may have different ideas at the different stages of growth. Therefore it is not enough to just conduct a demand survey, since it may ignore the timeliness of demand. At the same time, the demand survey with timeliness is more objective, which can be used for the reference to the early propaganda, so that it is able to better fit the students' ideas at different stages, and to produce more extensive and more effective influence. On the other hand, the understanding of students' feedback on the previous lecture is helpful to understand the students' periodic ideas, in order to make corresponding adjustments in the next lecture in a timely manner. Furthermore, it also facilitates students' exchange on the lecture, increases interaction, and spreads the content of the lecture for the second time. In this way, the value of the lecture will be able to be excavated for the second time in the student's thinking collision, which will improve the value and effect of education.

\subsection{To make a careful design of the content of the lecture and internalize students' motivation to attend the lecture}

The content of the lecture should be combined with students' actual situation and guide students to explore independently. ${ }^{9}$ First of all, students need more advanced knowledge. As we have entered the era of big data, the students' access to knowledge has widened greatly, the amount of information has increased several times, and the speed to get information has been rising. Therefore, the students have accordingly enhanced their standards for the demand of knowledge and other aspects, and the speed of enhancement has been accelerating. A large number of keynote speakers have misunderstood that they give a lecture on the theories and knowledge that the students have learned or are able to learn through the Internet, which can not meet the needs of the students at the moment, but satisfies their outdated needs, or the needs in the subjective thoughts of outsiders. Moreover, students hope that the content of the lecture is operable. The present is an era of information explosion. The theoretical information is instilled in large quantities at an excessively fast speed, which is unable to be fully integrated with reality. As a result, students can learn the theories from the textbooks in class, but they have only a few opportunities to put it into practice. Therefore, if the speaker wants to meet the needs of the students, it is necessary for him to 
accomplish two aspects. One is to teach more advanced and novel content, and the other is to teach highly practical knowledge.

\subsection{To create targeted lectures with high yield, guided by interactive experience.}

The post-1995 generations of college students have strong desire for knowledges. In addition to the content of lectures, forms, methods and means have had an important influence on the attitude of students towards attending lectures. In the traditional classroom teaching process, teachers are too innumerous to enumerate for having inevitably shown a certain sense of boredom and rigidity in teaching content. ${ }^{10}$ The form of lectures is more flexible and plastic than the traditional teaching forms. In accordance with the author's investigation, the new form of lecture that students are more inclined to is an interactive lecture, of which the salon is a form highly frequently mentioned by students. By asking the teachers in our school, we know that the school has held some salons, but not so many students as expected have attended the salons. The author finds that the lectures are unable to really attract students' attention by simply changing the forms.

Table 2: Types of lectures preferred to by students

\begin{tabular}{|l|c|}
\hline \multicolumn{1}{|c|}{ Option } & Proportion \\
\hline One-way instruction & $5.25 \%$ \\
\hline Form of salon & $20.13 \%$ \\
\hline Interviews with the guests & $11.49 \%$ \\
\hline Experience oriented & $40.06 \%$ \\
\hline Interaction oriented & $23.07 \%$ \\
\hline
\end{tabular}

Before the improvement of the form of lectures, it is necessary to have an in-depth understanding of the new forms of lectures and the nature of participating groups and to conduct a horizontal analysis of interest. Salon-oriented groups are relatively strong in community and change along with the change of the topic. If we want such a form to be sustainable, we need to stabilize this group and promote its internal flow. At the same time, it will be conducive for more students to having more chances to participate in it to hold the salons with the same topic but different contents for several times, which will form the internal flow of students. During the interactive discussion, one can explore ways to comprehending the knowledge of major. ${ }^{11}$ On the whole, regular salons with the unified topic will have a great many favorable possibilities to change the current situation, and it is one of the ways to solve the current situation. In the innovation of the form of lectures, we should take into account the nature of the form and the characteristics of the group oriented, which will help to improve the status quo.

\subsection{To add the finishing touch by technology, open up information channels, and create greater influence}

\subsubsection{To attach importance to value explanation and assist students in understanding the value of lectures}

In the propaganda of the lecture, the author believes that, in addition to the necessary information description, we should increase the interpretation of its value. Some students and teachers have attributed the poor attendance rate of lectures to the lack of early publicity. After investigation, it is found that the main channels for students to get information on lectures are notifications in class groups, official accounts on Weibo and WeChat, posters and students' notification. It mainly depends on the change in the development of the students themselves due to the lectures when most of the students choose whether to attend lectures or not. Therefore, the author suggests that we should overcome the shortcomings of traditional communication mode, such as narrow scope, small 
influence and single audience ${ }^{12}$. Besides the necessary information, the propaganda of the campus lectures should increase the writing of advertorials, and rise the content of the lecture itself to value realization of the behavior and consciousness, which will enable the students to have more intuitive feelings, to learn about the harvest that can be obtained from the lecture in advance and to attend the lecture based on their own needs.

3.4.2 To give lectures both online and offline to maximize the participation rate and influence of lectures

The Internet era has brought great impact to all walks of life, and lectures in universities are no exception. ${ }^{13}$ On the basis of the restrictions of the site capacity and the optimization of lecture results, there tends to be a limited number of students attending the lecture, so that some students may be willing to attend the lecture but they are unable to attend. The author suggested that the live broadcast system should be set up to carry out real-time online lectures, so that the students who can not attend the lectures have no regrets, but it is necessary to pay attention to communication with the keynote speaker, so as to avoid the disputes over copyright and other aspects. This function can be combined with the online expectation and feedback system, to take full advantage of the convenience from modern information technology, which will improve the effectiveness and influence of the lecture in its early, middle and later stages in an all-round way.

3.4.3 To realize resource sharing based on the support of macro policies and fine tutoring

The author suggests that both sides of the school and the college should establish smooth cooperative channels, and a transparent and standardized system of expenditure on lectures, ${ }^{14}$ and strengthen the integration of professional knowledge and popular content. For example, the law school can hold lectures on popularization of the laws, and the statistics school can hold lectures on learning methods of college mathematics. The college should strengthen the in-depth docking with the school, which help to improve the quality of the experts invited by taking advantage of the resources of the school, so that the students of this major are able to get into contact with the resources of other colleges, expand their horizons and improve their comprehensive qualities. In addition, it is necessary to gradually weaken the imbalance of high-quality education resources and to realize the effective sharing and transfer of resources through the mutual flow of lecture resources among different colleges and universities in the same area. ${ }^{15}$ Furthermore, we should establish and improve the information investment, management system and mechanism in education on a regional basis, ${ }^{16}$ so as to realize the effective utilization of resources, maximize the utility and promote the all-round development of the students.

\section{Conclusion}

In view of the impact of multiple lectures under the background of boundary change, the change of popular culture under the background of social change and the baptism of multiple cultures under the background of technological change, we need to consolidate the dominant position of the value concept of the college lectures, and strengthen the design sense of the cultural carrier so as to enhance the nutrient of the soil to cultivate the talents as pillars of the state, so that the socialist successors can be cultivated to rejuvenate and strengthen the country.

\section{References}

[1] J Wang Yaping and Zheng Qiujuan, Influence of Campus Lectures on Humanistic Quality Education among Collect Students Majoring in Science and Engineering -Based on Case Study of Humanities Lectures for College Students in Jiangsu University of Technology, Journal of Jiangsu University of Technology, vol. 20, pp100-104, 2014.

[2] J Zeng Jianxiong, Song Dan, and Gao Shuren, Study on the College Students' the Second Classroom: Course, Focus and Prospect_-A Literature Review Based on the CNKI Literatures from 1999 to 2016 , Chongqing Higher Education Research, vol. 5, pp119-127, 
2017.

[3] M Fugate, A. J. Kinicki, and B. E. Ashforth, Employability: A psycho-social construct, its dimensions, and applications, Journal of Vocational Behavior, vol.1, pp65, 2004.

[4] J Jiayi Lu. College Students' Attending the Lectures: Significance, Current Situation and Countermeasures, Jian nan Wenxue, vol. 5, pp 240-241, 2013.

[5] M Peter F.Drucker, Personal management, Shanghai University of Finance and Economics Press, pp268, 2003.

[6] J Chen Mei, Exploration into "Overall Ideological and Political Education Mode" for the Ideological and Political Campus Lectures in Colleges and Universities, Heihe Journal, vol. 2, pp 103, 2015.

[7] J Tong Jiaojiao and Wang Yulu, Conditions and Way out for the College Lectures Based on Information Challenges, College Advisor, vol. 4, pp55-56, 2013.

[8] J John H. Van de Graaff, Academic Power-Patterns of Authority in Seven National Systems of Higher Education, Hangzhou: Zhejiang Education Publishing House, pp139, 2001.

[9] Meng Fang, Qiu Yuxing, and Chen Zhenyu et al, Research on Curriculum System Based on the Innovative Personnel Training Mode in Colleges and Universities, Business Economy, vol. 9, pp 118-120,2012.

[10]Fan Jiebing, Research on the Construction of Lecture System Based on the Cultivation of Innovative Talents in Colleges and Universities, Journal of Changchun University, Vol27 pp43-45,2017.

[11]M Huang Junjie, University general education in the era of globalization, Peking University Press, pp 94, 2006.

[12]J Ouyang Qiannan, Research and Solutions to Information Asymmetry in Lectures, Person Education, vol. 10, pp88-89,2014.

[13]J Xia Shouxin and Zhang Jiajun, College Lectures in the Internet Age, Decision \& Information, vol. 2, pp148, 2016.

[14]J Wang Junfang, Existing Problems in Academic Lectures in Universities and Suggestions for Improvement, Education Space, vol. 14, pp 162, 2013.

[15]J Ren Ke, On the Sharing Path of Lecture Resources in Regional Colleges and Universities, Educational Review, vol. 3, pp24-25,2014.

[16]M Allen Kent, The Goals of Resource sharing in Libraries, see://KentA,Galvin TJ. Library Resource Sharing: Proceedings of the 1976 Conference on Resource Sharing in Libraries. Pittburgh: New York: Mareel Dekker, Inc, pp 18,1977. 